\title{
L'hétéroglossie littéraire dans Illusions perdues de Balzac: Enjeux, fonctions et limites
}

\author{
Literary heteroglossia in Balzac's Illusions perdues: Issues, \\ functions and limits
}

\author{
Heteroglossia literária nas ilusões perdidas de Balzac: razões, \\ funções e limites
}

Mohamed Bourasse*

mohamed.bourasse@uit.ac.ma

Faculté des Lettres et Sciences humaines à Kénitra

RÉSUMÉ : A lire Illusions perdues, le premier constat qui frappe le lecteur, est l'extrême diversité du langage de ses personnages. Balzac ouvre sa langue littéraire sur une infinité de discours oraux et écrits, de nature familière, régionale, diachronique.... Et ce, par l'usage d'une palette diverse de variantes linguistiques, se répartissant en sociolectes, idiolectes, régiolectes..., phénomène que certains chercheurs appellent "l'hétéroglossie ${ }^{1}$ littéraire". Tous les types de variation parsèment le roman et la langue balzacienne n'est pas homogène et monolithique, mais hétérologique et hétéroglossique. Notre objectif dans cette réflexion est de problématiser cette notion d'hétéroglossie littéraire dans une perspective littéraire et sociolinguistique (variationniste). Pour ce faire, nous étudierons les raisons et les motivations de cette pratique, ainsi que ses effets et ses fonctions. Nous examinerons également les écueils que ce phénomène peut produire dans l'œuvre.

LES MOTS CLÉS : Hétéroglossie littéraire. Balzac. Illusions perdues. Variation linguistique.

ABSTRACT: To read Illusions perdues, the first observation that strikes the reader is the extreme diversity of the language of its characters. Balzac opens his literary language on an infinity of oral and written speeches, of a familiar, regional, diachronic nature ... And this, by the use of a diverse palette of linguistic variants, divided into sociolects, idiolects, regiolects ..., a phenomenon that some researchers call "literary heteroglossia". All types of variation dot the novel and the Balzacian language is not homogeneous and monolithic, but heterological and heteroglossic. Our objective in this reflection is to problematize this notion of literary heteroglossia from a literary and sociolinguistic (variationist) perspective. To do this, we will study the reasons and

\footnotetext{
* Doctorant à la faculté des Lettres et des Sciences humaines à Kénitra (Maroc), Laboratoire Langage et Société. /Aluno de doutorado na Faculdade de Letras e Ciências Humanas de Kénitra (Marrocos), Laboratório de Linguagem e Sociedade. /PhD student at the Faculty of Letters and Human Sciences in Kénitra (Morocco), Language and Society Laboratory.

${ }^{1}$ Terme de Rainier Grutman. In : Grutman, R. Des langues qui résonnent. L'hétérolinguisme au XIXe siècle québécois. Montréal : Fides/Cétuq, 1997.
} 
motivations for this practice, as well as its effects and functions. Also, we will examine the pitfalls that this phenomenon can produce in the work.

KEYWORDS: Literary Heteroglossia. Balzac. Illusions perdues. Linguistic variation.

RESUMO: Para ler Illusions perdues, a primeira observação que impressiona o leitor é a extrema diversidade da linguagem de seus personagens. Balzac abre sua linguagem literária em uma infinidade de discursos orais e escritos, de natureza familiar, regional, diacrônica ... $E$ isso, pelo uso de uma paleta diversificada de variantes lingüísticas, divididas em socioletos, idioletos, regioletos ..., fenômeno que alguns pesquisadores chamam de "heteroglossia literária". Todos os tipos de variação pontuam o romance, e a linguagem balzaciana não é homogênea e monolítica, mas heterológica e heteroglóssica. Nosso objetivo nesta reflexão é problematizar essa noção de heteroglossia literária a partir de uma perspectiva literária e sociolingüística (variacionista). Para isso, estudaremos os motivos e motivações dessa prática, bem como seus efeitos e funções. Além disso, examinaremos as armadilhas que esse fenômeno pode produzir no trabalho.

PALAVRAS CHAVE: Heteroglossia literária. Balzac. Illusions perdues. Variação linguística.

\section{Introduction}

La présente étude s'inscrit dans un projet plus large qui interroge la relation dichotomique langue/littérature, voire tripartite langue/littérature/société, ainsi que les interactions entre elles et la question du service que l'une rend à l'autre. En effet, les frontières entre langue, littérature et société sont si fines et subtiles que nous ne pouvons pas les séparer. C'est pour cela que plusieurs disciplines telles que la stylistique, la linguistique de l'énonciation, la sociocritique se sont penchées sur ce rapport langue/ littérature, voire sur la variation linguistique au sein des textes littéraires. Notre corpus ${ }^{2}$ d'analyse a porté sur un roman de Balzac Illusions perdues, l'une des œuvres majeures de son immense projet littéraire La Comédie humaine. L'extrême diversité du langage de ses personnages nous pousse étudier cette œuvre de Balzac. Ainsi, contrairement à certains écrivains classiques qui couvrent la voix de leurs personnages d'une langue littéraire monolithique et monologique, Balzac ouvre sa langue sur une infinité de discours oraux et écrits, de nature familière, régionale, diachronique... Et ce, par l'usage d'une palette très diverse de variantes linguistiques, se répartissant en sociolectes, idiolectes, régiolectes..., phénomène que certains chercheurs se plaisent à appeler "l'hétéroglossie littéraire". Tous les types de variation

\footnotetext{
2 BALZAC, Honoré de. Illusions perdues. Paris : Ed. Gallimard, 2013.
} 
parsèment le roman, touchant tous les niveaux linguistiques avec une nette saillance du niveau lexical. Notre étude débouche sur un ensemble de constats: la langue balzacienne n'est pas homogène et monolithique, mais hétérologique et hétéroglossique. Le lecteur y rencontre les sociolectes de la bourgeoisie, de l'aristocratie, des paysans... ; les régiolectes de la Province, de Paris, de l'Alsace... A cela, s'ajoutent quelques idiolectes atypiques de certains personnages comme Kolb et le père Séchard.

Tout cela nous pousse à nous interroger sur les raisons et les enjeux littéraires et linguistiques qui sont à l'origine de cette pratique littéraire chez Balzac. En d'autres termes, qu'est-ce qui motive cette pratique chez l'auteur par opposition aux autres écrivains qui recouvrent les paroles de leurs personnages d'une langue normée, soutenue et monolithique ? Ce phénomène était l'objet de maintes études, et Rainier Grutman, entre autres, est l'un des critiques littéraires intéressés par cette pratique ; il la qualifie d'hétérolinguisme littéraire, qu'il définit ainsi : " La présence dans un texte d'idiomes étrangers, sous quelque forme que ce soit, aussi bien que de variétés (sociales, régionales ou chronologiques) de la langue principale » (GRUTMAN, 1997, p. 37). Concernant la même notion, il ajoute ailleurs que « l'hétérolinguisme textuel désigne la textualisation d'idiomes en littérature, qu'il s'agisse de véritables langues étrangères ou seulement de variétés particulières de la langue principale de l'œuvre » (GRUTMAN, 1997, p. 91).

L'objectif de cette réflexion est de problématiser cette notion d'hétérolinguisme littéraire ou autrement nommée " l'hétéroglossie littéraire », à travers une perspective énonciative, sociolinguistique et narratologique pour rendre compte de la textualisation de la variation linguistique dans Illusions perdues. Certes, le choix de Balzac de cette langue d'écriture ne peut pas être anodin. En effet, les exemples que nous avons étudiés dans cette œuvre montrent bien que l'introduction de la variation dans la langue littéraire ne peut d'aucune façon être considérée comme un simple procédé à valeur ornementale et sans effet sur la dynamique de l'œuvre, comme en témoigne le point de vue de Lise Gauvin qui a affirmé à cet égard :

La problématique des interactions langues / littératures est complexe et met en cause aussi bien des enjeux institutionnels - l'autonomie d'une littérature, les conditions de son émergence, la relation qui s'y établit entre l'écrivain et le public -, politiques - le statut d'une langue et de ceux qui la parlent -, que proprement littéraires, à savoir quels sont les modèles dont dispose l'écrivain pour représenter les rapports 
sociaux entre les langues ou les niveaux de langue (GAUVIN, 1999, p. 53).

Ainsi, loin de toute exhaustivité, tant s'en faut, nous nous en tiendrons ici aux raisons et motivations qui nous paraissent comme étant déterminantes et essentielles, ainsi que les effets et les fonctions de cette pratique au sein de l'œuvre littéraire. Au terme de cette réflexion, on s'attardera sur quelques écueils et problèmes que ce phénomène peut produire.

\section{Raisons et enjeux de la variation chez Balzac}

\subsection{L’impact du réalisme sur la langue de Balzac}

En tant que mouvement littéraire du $\mathrm{XIX}^{\mathrm{e}}$ siècle, le réalisme se traduit par la volonté de certains écrivains romanciers de représenter le plus fidèlement possible le monde réel. Cette esthétique romanesque est venue avec un certain nombre d'écrivains comme Balzac, Zola, Maupassant..., comme réaction contre le romantisme pour s'élever contre ses idéalisations excessives de la réalité. II se caractérise par la volonté de représenter dans l'œuvre littéraire la réalité dans toutes ses dimensions sociales, culturelles, économiques... Balzac, comme nous l'avons vu dans Illusions perdues, ne s'arrête pas là, il s'efforce de reproduire le plus fidèlement possible les multiples types de discours de ses personnages. Ainsi la technique réaliste de Balzac ne passe-t-elle pas seulement par la description de lieux, d'objets et de personnages, ou ce que Philippe Hamon appelle le réalisme descriptif (1973, p. 416), mais également par la reproduction de différents types de variétés linguistiques qui sous-tendent la réalité linguistique des personnages de l'époque. Type de réalisme que Philippe Hamon appelle le réalisme textuel (1973, p. 416), ou linguistique. Ce type de réalisme contribue même plus que le réalisme descriptif à l'effet du réel dans la mesure où les discours mis dans la bouche des personnages sont les mêmes que ceux de la réalité, tandis que les lieux, les objets, les personnages décrits par le narrateur sont toujours fictifs.

Par l'incorporation de cette variation linguistique dans Illusions perdues à travers la stratification du langage de ses personnages, Balzac pousse sa technique réaliste plus loin et accroît le degré de vraisemblance de l'œuvre dans son ensemble. II donne ainsi une valeur ajoutée à la représentation vraisemblable de la réalité qui est toujours hétéroglossique et plurilingue. Ainsi, l'importance qu'il accorde à la vraisemblance, but 
ultime de toute technique réaliste, le conduit à prêter une attention minutieuse à la langue utilisée par ses personnages et son narrateur omniscient lui-même, pour la reproduire le plus fidèlement possible. Chose qui justifie le recours massif au discours direct par l'auteur, car le changement de langue ne peut s'opérer que lorsqu'il y a changement de locuteurs au cours du dialogue. En effet, le discours direct est le type de discours rapporté que Gérard Genette considère comme étant le plus mimétique. II a le moins de distance car le narrateur cède la parole aux personnages, comme l'ont confirmé Denis Labouret et André Meunier en affirmant que « dans le récit, le discours direct est censé restituer fidèlement la vivacité, l'expressivité des propos : il donne à entendre la voix même des personnages » (LABOURET; MEUNIER, 2004, p. 40).

Au sujet de la technique réaliste de Balzac ou le réalisme linguistique, Erich Auerbach (1968) déclare que Balzac a introduit un nouveau mode de vraisemblance réaliste dans lequel, précisément, la mimesis linguistique, à côté de la mimesis descriptive, occupe une place prééminente. Pour lui, ce réalisme est un mélange de registres et de styles car les personnages parlent leur langue sans aucune stylisation littéraire. Le rejoint en cela Sainte Beuve qui voit dans l'écriture de Balzac « la marque du réalisme, une prose toute en diversité linguistique, tant par la voix du narrateur que par la parole des personnages » (Sainte-Beuve,1883, p. 394).

D'après l'étude d'lllusions perdues, il semble aller de soi que Balzac a poussé sa mimesis linguistique plus loin, il s'efforce ainsi de reproduire le parler de ses personnages dans toute sa diversité jusqu'à même la prononciation déformée, comme celle de Kolb qui débouche sur l'incompréhension du lecteur. Car Balzac est fort conscient de l'importance de la prononciation dans la caractérisation populaire de ses personnages, si bien qu'il est amené à souligner soigneusement l'idiolecte de Kolb, non pas au moyen de termes particuliers, mais par le biais d'une transcription phonétique fidèle :

-Si matame feut addentre ein bedit quard'hire, che fais bousser eine regonnaissanze dans le gampe ennemi, dit Kolb, et vis ferrez que che m'y gonnais, quoique chaie l'air d'ein Hallemante ; gomme che suis ein frai Vrançais, chai engor te la malice. ${ }^{3}$ (BALZAC, 2013, p. 675)

Pour conclure, nous pouvons dire que l'un des piliers fondamentaux de la technique réaliste de Balzac passe précisément par la reproduction fidèle des parlers de

\footnotetext{
${ }^{3}$ L'italique par Balzac
} 
ses personnages. Chose qui fait de la langue balzacienne une véritable source de vraisemblance. Philippe Hamon (1973, p.416), quant à lui, la compte parmi les quinze marqueurs qui contribuent au réalisme global de l'œuvre littéraire. Ce souci linguistique avoué par Balzac est lié chez lui au concept général de la mimésis aristotélicienne pour répondre aux exigences du courant réaliste du $\mathrm{XIX}^{\mathrm{e}}$ siècle. La langue d'écriture et le réalisme sont ainsi étroitement liés. Ce qui fait d'Illusions perdues un ensemble romanesque hétéroglossique et hétérologique.

\subsection{Une esthétique d'écriture}

Si le souci du réalisme est la raison principale de l'incorporation de la variation linguistique dans l'œuvre de Balzac, ou autrement nommée l'hétéroglossie littéraire, cette dernière possède d'autres connotations que nous pouvons interpréter cette fois dans une perspective purement esthétique. Ainsi la stratification des langages des personnages dans Illusions perdues reste-t-elle à lire comme étant une esthétique singulière de l'écriture balzacienne, que nous pouvons associer à la première raison de la mimésis linguistique, puisque l'une débouche sur l'autre. Sur ce point, Roman Jakobson considère le réalisme en littérature une question de convention esthétique (1973, p. 31-39), invoquée par une génération d'écrivains pour se démarquer d'une génération précédente, qui se présente comme une sorte de métonymie d'une société moderne éclectique. Dans son sillage, Erich Auerbach (1968) voit parmi les caractéristiques de cette esthétique réaliste un mélange de registres et de langages.

Loin d'être un procédé d'écriture anodin et sans effet, cette hétéroglossie est vue par les critiques comme une nouvelle esthétique de la littérature du XIXe siècle. $A$ cet effet, nous pouvons citer le point de vue de Bakhtine qui y voit une esthétique indispensable à l'art du roman en affirmant que « le roman pris comme un tout, c'est un phénomène pluristylistique, plurilingal, plurivocal »(BAKHTINE, 1978, p. 87). II ajoute ailleurs :

Le style du roman, c'est un assemblage de styles; le langage du roman, c'est un système de langues (BAKHTINE, 1978, p. 88).

Le roman, c'est la diversité sociale de langages, parfois de langue et de voix individuelles, diversité littérairement organisée. Ses postulats indispensables exigent que la langue nationale se stratifie en dialectes sociaux, en maniérismes d'un groupe, en jargons professionnels, 
langages des genres, parler des générations, des âges, des écoles, des autorités, cercles et modes passagères, en langages des journées (voire des heures) sociales, politiques (chaque jour possède sa devise, son vocabulaire, ses accents); chaque langage doit se stratifier intérieurement à tout moment de son existence historique (BAKHTINE, 1978, p. 88).

Pour Bakhtine, la variation linguistique est une esthétique inhérente à l'art du roman qui n'est jamais pur mais, au contraire, hétérogène, composite, et est le produit d'un alliage de voix. Dans le même sens, Barthes, parlant du texte littéraire en général, observe que « le texte est d'emblée, en naissant, multilingue » (BARTHES, 1975, p. 127). En tant qu'esthétique littéraire du XIXe siècle, le degré de cette variation linguistique varie selon les genres littéraires. Le genre poétique est celui qui, comme l'écrit Bakhtine, tend le plus vers le monologisme car l'unité du système du langage et l'unité de l'individualité linguistique sont les règles indispensables du style poétique (BAKHTINE, 1978, p. 90). Le roman, au contraire, se caractérise par son hétérogénéité et son caractère hybride, par la stratification interne des langages sociaux et la diversité des voix qui y résonnent.

De son côté, Théophile Gautier, dans son étude sur Balzac, a remarqué ce changement ou cette particularité dans la prose narrative de l'auteur. II associe cela à la modernité, car, pour lui, être historien de la réalité contemporaine suppose une langue spéciale. Une réalité qui se caractérise par l'éclatement de l'être et la société, et par l'hybridité. Devant l'éclatement et la disparité de sa société, Balzac se voit confronté au besoin d'inventer une langue capable de mieux décrire cette réalité :

[...] Pour exprimer cette multiplicité de détails, de caractères, de types, d'architectures, d'ameublements, Balzac fut obligé de se forger une langue spéciale, composée de toutes les technologies, de tous les argots de la science, de l'atelier, des coulisses, de l'amphithéâtre même. Chaque mot qui disait quelque chose était le bienvenu, et la phrase, pour le recevoir, ouvrait une incise, une parenthèse, et s'allongeait complaisamment (GAUTIER, 1999, p. 289).

En guise de conclusion, nous pouvons dire que l'hétéroglossie littéraire est un choix stratégique résultant d'une tendance esthétique qui oriente la dynamique globale de l'œuvre de Balzac. En effet, écrire c'est un travail de langage et le choix de telle ou telle langue d'écriture est révélateur d'une esthétique littéraire. Balzac prend dans Illusions perdues la variation linguistique comme un support pour forger son esthétique littéraire. II veut rompre avec l'idéal de la langue littéraire classique qui est homogène, 
monologique et soutenue, pour construire une littérature éclectique de l'écart qui laisse le lecteur vivre l'expérience de l'écart par rapport à la langue uniforme et soignée, à laquelle la littérature classique et traditionnelle l'a habitué. Pour lier cette raison à la précédente, nous pouvons dire que la vraisemblance du discours littéraire de Balzac se fonde principalement sur une esthétique linguistique d'écriture.

\subsection{L'environnement sociolinguistique d'Illusions perdues}

Nous allons aborder ici une autre raison que nous ne pouvons pas dissocier de la première : l'environnement sociolinguistique de la production. Tout le monde s'accorde sur l'impossibilité pour un écrivain de se départir de son univers socioculturel et linguistique. La littérature reste toujours imprégnée de la réalité extra-littéraire de sa production et en porte les marques. Ainsi, l'hétéroglossie littéraire dans Illusions perdues qui se manifeste à travers la stratification du langage des personnages nous pousse à repenser la relation entre la littérature et la réalité. Dans l'œuvre de Balzac, cette stratification constitue le miroir de la dynamique du français en France du XIXe siècle. La réalité linguistique du français s'infiltre dans Illusions perdues à travers la des personnages de Balzac.

Nous ne pouvons pas parler de l'environnement linguistique où l'œuvre de Balzac est apparue sans parler de la politique linguistique de la France. Cette dernière est l'un des premiers Etats occidentaux à avoir établi et maintenu un ensemble de dispositifs visant à homogénéiser les pratiques linguistiques sur son territoire depuis le XVII avec la naissance de l'Académie française, dont la tâche était de donner les règles et de purifier la langue des patois et des régionalismes. Elle travaille ainsi intensément à l'établissement de la norme. Le début de la révolution française se montre centralisateur et va dans le sens de l'uniformisation de la langue française. Les révolutionnaires tentent de restreindre les langues autochtones pour imposer le français normé comme une langue nationale, institutionnelle et administrative.

Pourtant, la politique linguistique sous le premier Empire marque un retour à une politique de laisser-faire en ce qui concerne les langues régionales. La répression de la diversité est abandonnée. Aussi, avec Napoléon, l'Etat revient indirectement sur l'obligation d'enseigner en français. Précisément à cette époque, les dialectes s'épanouissent grâce à l'exode rural et l'industrialisation, malgré la politique linguistique de l'Etat qui repose sur l'unification, comme l'a souligné Sainte Beuve : 
Le moment actuel est, à certains égards, tout l'opposé de celui de Vaugelas. Alors tout tendait à épurer et à polir ; aujourd'hui tout sembler aller en sens contraire, et un mouvement rapide d'intrusions se manifeste. Alors tous les mauvais mots demandaient à sortir ; aujourd'hui tous les mots plébéiens, pratiques, techniques, aventuriers même, crient à tue-tête et font violence pour entrer (Sainte-Beuve, 1883, p. 394).

Alors la littérature de cette époque est influencée par la situation de la diglossie, voire de l'hétéroglossie, qui sous-tend la scène linguistique de la France du XIX ${ }^{\mathrm{e}}$ siècle, où les dialectes commencent à acquérir une grande dignité sociale, au point qu'ils n'étaient pas seulement employés par les couches les plus basses, mais par les classes les plus cultivées et lors des occasions officielles. Ces dialectes ont même trouvé leur place dans la communication écrite et ont ouvert le chemin à une littérature en dialectes. Cette littérature réaliste est surement venue contre le romantisme monologique et national, qui ne correspond plus à la réalité linguistique fracturée et éclatée du $\mathrm{XIX}^{\mathrm{e}}$ siècle, comme l'a affirmé Bakhtine qui affirme que « si les principales variantes des genres poétiques se développent dans le courant des forces centripètes, le roman et les genres littéraires en prose se sont constitués dans le courant des forces décentralisatrices et centrifuges ॥ (BAKHTINE, 1978, p. 96). De ce fait, pour décrire la société postrévolutionnaire entrant dans l'industrialisation, cette langue littéraire classique n'est plus de mise. Lise Gauvin reconnait l'influence de l'environnement sociolinguistique sur l'écrivain en affirmant :

[...] La situation des écrivains francophones est emblématique d'un parcours qui les condamne, de quelque lieu qu'ils proviennent, à penser la langue. La proximité des autres langues, la situation de diglossie sociale dans laquelle ils se trouvent le plus souvent immergés, une première déterritorialisation constituée par le passage de l'oral à l'écrit, et une autre, plus insidieuse, créée par des publics immédiats ou éloignés, séparés par des acquis culturels et langagiers différents, sont autant de faits qui les obligent à énoncer des stratégies de détours (GAUVIN, 1999, p. 10).

Bref, nous pouvons dire que Balzac a écrit à une époque où le français populaire se fond peu à peu dans la langue écrite nationale et institutionnelle. En effet, cette dernière commence à cette époque à se stratifier intérieurement en sociolectes, en régiolectes..., comme il est constaté par Balzac lui-même : « aujourd’hui notre langue a 
autant d'idiomes qu'il existe de variétés d'hommes dans la grande famille française » (BALZAC, 1832, p. 142). Edouard Glissant, de son côté, voit que la littérature part toujours du contexte linguistique de la production en précisant qu'« on ne peut plus écrire une langue de manière monolingue. On est obligé de tenir compte des imaginaires des langues » (GAUVIN, 1996, p. 112). En conséquence, c'est cette situation d'hétéroglossie qui met des écrivains comme Balzac devant un choix difficile entre, d'une part, la langue standard hautement considérée dans la société mais fort éloignée de la réalité des personnages, et d'autre part, la langue populaire vernaculaire parfois à tradition orale, celle du milieu social des personnages.

En guise de conclusion, la situation d'hétéroglossie de la France du XIXe siècle, mène Balzac à s'exposer au phénomène de plurilinguisme textuel et à textualiser parodiquement cette diversité linguistique dans Illusions perdues à travers l'incorporation de tous les types de variation linguistique. II essaye de s'approprier le vernaculaire, longtemps abandonné, et de lui rendre sa dignité littéraire, en en faisant une langue littéraire hybride et hétérologique qui est « une construction à l'intérieur de la langue commun », comme l'a souligné Lise Gauvin (1999, p. 54). Tout se passe ainsi comme si Balzac voulait rendre floues les frontières qui séparent la langue normée classique et les variétés vernaculaires. Pour Lise Gauvin, cette mise en scène de la variation linguistique sous-tendant l'environnement linguistique de l'écrivain, Balzac en l'occurrence, au sein de l'œuvre littéraire, est un indice de la naissance d'une certaine «surconscience linguistique » qui se développe chez cet écrivain (GAUVIN, 1999, p. 172).

\section{Fonctions et limites de l'hétéroglossie dans Illusions perdues}

\subsection{Fonctions et effets de l'hétéroglossie littéraire}

Comme nous l'avons vu plus haut, le recours de Balzac à l'hétéroglossie littéraire ne peut pas être une pratique anodine et fortuite. En effet, l'incorporation de la variation linguistique dans Illusions perdues est une stratégie singulière et subtile de la technique réaliste de Balzac que nous devons interpréter dans une perspective littéraire, puisque notre corpus est une œuvre littéraire. La présence de cette variation revêt plusieurs fonctions et elle est utilisée à des fins très diverses selon les écrivains. Pour Balzac, cette pratique remplit une fonction avant tout réaliste et référentielle. Ainsi, pour mieux répondre aux exigences du réalisme, il a introduit la variation linguistique dans toutes ses formes dans son œuvre pour accroitre la vraisemblance de l'histoire, son but premier et 
ultime, et produit ainsi l'illusion de vrai. La présence de cette variation se présente comme une dose supplémentaire du réalisme que Balzac injecte dans l'histoire. Comme nous l'avons vu supra, c'est la plus apte à accroitre le degré de vraisemblance de l'œuvre que les autres techniques. Autrement dit, elle produit au sein de l'œuvre 'l'effet de réel" que Roland Barthes définit comme étant « la vraisemblance inavouée qui forme l'esthétique de toutes les œuvres courantes de la modernité »(BARTHES, 1982, p. 89).

Par ailleurs, ce faisant, l'auteur confère une couleur locale à son histoire. Et ce, en délégant la parole à ses personnages pour inclure des idiolectes et des sociolectes les plus représentatifs de la société de son époque, pour donner à entendre les parlers de son temps. En outre, les régionalismes et les diachronismes ancrent l'histoire dans un espace et un temps bien précis (la France -Paris et la Province- sous la Restauration). Parfois, Balzac cherche des idiomes atypiques qui échappent à la langue commune. C'est peut-être la raison d'apparaitre d'un personnage secondaire comme Kolb, atypique par sa prononciation déformée et défectueuse. Chose qui permet de saisir à travers la parole des personnages les langages de cette époque, que l'auteur sollicite tous. Toute cette diversité linguistique fait du roman une société miroir de la société réelle, un milieu extra-littéraire à tous les niveaux. Lise Gauvin reconnait évidemment l'effet de cette variation linguistique quand il a précisé que les effets de langue deviennent des effets de réel.

Outre cette fonction référentielle, l'hétéroglossie littéraire remplit une fonction purement sociolinguistique. Dans cette optique, le but principal de cette pratique est de faire correspondre les personnages de Illusions perdues avec leur classe sociale, leur milieu géographique, leur époque... La manière de parler d'un personnage peut nous renseigner sur son appartenance et son environnement. En effet, chacun est renvoyé donc par sa pratique linguistique à son origine. Nombreux sont les cas dans Illusions perdues où rien ne nous renseigne sur les personnages que leur manière de parler (cas de Kolb, Florentine, le père Séchard...). Elle permet aussi de distinguer le parler de la Province de celui de Paris, en présentant le premier comme plus relâché et le deuxième comme plus soigné. Outre cette caractérisation sociale et régionale, les diachronismes parsemant Illusions perdues ancrent l'histoire dans la France postrévolutionnaire.

Cette pratique remplit en outre une fonction linguistique et idéologique. Cette fonction témoigne de la volonté de Balzac de redonner aux dialectes de son temps leur dignité sociale et leur valeur linguistique, suite aux efforts de l'Etat de l'uniformisation et son attaque aux patois. Cette variation linguistique se conçoit donc comme un rempart 
pour endiguer la standardisation et l'unification linguistique prônée par l'Etat de son temps. Pour ce faire, Balzac s'efface derrière ses personnages auxquels il délègue la parole pour faire entendre les parlers de son époque. Il prend ainsi cette pratique comme une stratégie subtile pour rendre compte de la mixité linguistique de la France d'alors. D'autre part, par cette pratique, Balzac élabore la philologie des variétés de sa société : il les recense, montre leur divergence, leur évolution, leur vitalité ou leur usure. Aussi, il met en évidence les tensions linguistiques produites par la situation sociolinguistique de son époque pour les neutraliser par la narration par la suite, et il rend compte des forces décentralisatrices et centrifuges du langage, comme l'a précisé Mikhaïl Bakhtine :

La catégorie du langage unique est l'expression théorique des processus historiques d'unification et de centralisation linguistique, des forces centripètes du langage. Le langage unique n'est pas donné, mais en somme, posé en principe et à tout moment de la vie du langage il s'oppose au plurilinguisme [...] le roman et les genres littéraires en prose se sont constitués dans le courant des forces décentralisatrices et centrifuges (BAKHTINE, 1978, p. 95-96).

Le point de vue de Rainier Grutman n'est pas différent du précédent, il voit, lui aussi, que "la diglossie littéraire est une dynamique d'écriture visant à dédramatiser les conflits linguistique" (GRUTMAN, 1997, p. 61).

Reste à parler de la fonction esthétique et littéraire de cette pratique. L'introduction de la variation écarte plus Balzac de son langage, en cédant la parole à ses personnages. Ce faisant, Balzac, contrairement à certains écrivains qui recouvrent la parole de leurs personnages par une langue littéraire soutenue, transgresse l'idéal de la langue littéraire classique en inventant sa langue propre qui vise le renouvellement de la langue traditionnelle habituelle dans les œuvres littéraires classiques. Balzac prend ainsi l'hétéroglossie littéraire comme le support de son esthétique d'écriture qui dissimule ses intentions et ses attitudes envers la situation linguistique de son époque.

Pour conclure, nous pouvons dire que Balzac, pour décrire la réalité en tout vérisme, se voit confronté au besoin de se forger une langue particulière, car la langue littéraire traditionnelle ne peut plus correspondre à la réalité éclatée et hybride de la situation linguistique de la France sous la Restauration. Une langue qui peut rendre compte de l'éclatement et de l'hybridisme linguistique qui caractérise l'époque de Balzac, que Philippe Hamon définit ainsi : 
Pas de lieu qui ne soit parloir : de nouvelles parlures, fortement localisées, s'installent, liées à la ville, en littérature : l'argot des basfonds, la blague de l'atelier, le bon mot du boulevard, les rumeurs de la Bourse, le barbarisme des loges, le cliché du salon bourgeois, [...] De nouveaux schèmes s'actualisent [...] comme un nouveau personnel littéraire conditionné par ces mêmes schèmes : le flâneur, le badaud, le gamin de Paris, le provincial à l'Exposition, la grisette, le bousingot, le rentier inspecteur de grands travaux, le bourgeois, le détective expert en filatures, la passante, le chiffonnier, le dandy, la mondaine et la fille des rues (HAMON, 1994, p.7).

\subsection{Limites et écueils de l'hétéroglossie littéraire}

Après nous être arrêté sur la présence de la variation linguistique dans Illusions perdues de Balzac, les fonctions qu'elle remplit au sein de l'œuvre, nous avons constaté que l'introduction de cette variation dans l'œuvre ne passe pas sans problèmes. Notre objectif ici est de nous arrêter sur quelques problèmes que l'hétéroglossie littéraire produit, ou les limites qui l'empêchent de remplir pleinement ses fonctions. Ainsi, pour la commodité de l'analyse, nous allons répartir ces problèmes en plusieurs niveaux.

Sur le plan littéraire, l'incorporation de la variation linguistique dans le roman de Balzac nuit à la littérarité de l'œuvre. En effet, si nous pouvons définir cette dernière par le degré d'importance que l'écrivain accorde à la beauté du style, et ce par l'usage de différents procédés d'écriture qui embellissent et polissent le style, la langue de Balzac est celle de tous les jours, celle de la conversation, privée de toute élaboration particulière. Bien entendu, nous pouvons lui attribuer une fonction référentielle et réaliste dans la mesure où elle reproduit le plus fidèlement possible les parlers d'une époque donnée, mais cela peut amoindrir sa portée poétique et littéraire. La langue de Balzac se présente alors comme un discours courant, neutre par rapport à l'art. Elle gagne en réalisme ce qu'elle perd en littérarité. Sur ce point, Boris Tomachevski a affirmé :

Le matériau réaliste ne représente pas en soi une construction artistique et, pour qu'il le devienne, il faut lui appliquer des lois spécifiques de construction artistique qui, du point de vue de la réalité, sont toujours des conventions (HAMON, 1973, p. 416).

Si nous revenons au schéma de communication de Jakobson, nous pouvons dire que le discours de Balzac est un discours référentiel par excellence, caractérisé par la priorité donnée à la fonction référentielle et à l'expression du référent, en négligeant en 
contrepartie la fonction poétique. Auerbach voit, lui aussi, que le texte devient naturellement plus parfait qu'il s'éloigne de l'archétype réaliste. A observer ce qui se passe dans la langue de Balzac par rapport à la langue littéraire classique, il est difficile de lui donner un statut poétique et littéraire. Devant ce dilemme, la question que nous pouvons paradoxalement poser est: peut-on parler d'un discours à la fois purement littéraire et réaliste ?

Le deuxième écueil touche cette fois à la fonction sociolinguistique de l'hétéroglossie littéraire et l'empêche d'être pleinement remplie. Nous avons vu plus haut comment cette pratique permet de faire correspondre les parlers des personnages à leur appartenance sociale, géographique ou autre. En revanche, la variation linguistique ne constitue pas toujours un marquage pertinent pour identifier socialement ou géographiquement les locuteurs dans la mesure où un même trait linguistique peut correspondre à plusieurs types de variation, comme l'a souligné Françoise Gadet :

\begin{abstract}
II est maintenant souvent difficile de localiser un locuteur à simple écoute, des facteurs sociaux comme la mobilité, l'éducation et les médias ayant eu des effets à la fois homogénéisants (entre variétés proche) et hybridisants (entre idiomes). Les particularismes locaux se maintiennent surtout quand les contacts sont limités: dans les campagnes, chez les âgés et les moins éduqués (Gadet, 2007, p. 15).
\end{abstract}

D'autre part, les registres de langue ne sont pas des groupes étanches nettement tracés. En effet, nombreux sont les phénomènes de langue qui peuvent être le fait d'un locuteur très cultivé, ou plutôt d'un locuteur ordinaire en situation officielle. Chose qui neutralise plus ou moins l'effet de la fonction sociolinguistique de la variation linguistique au sein d'Illusions perdues. Sans oublier la dimension diaphasique de la variation qui œuvre dans ce sens. C'est le cas, par exemple, de Lucien entre autres, qui oscille entre un provincialisme dont il ne parvient pas à se débarrasser (désangoulêmer ${ }^{4}$ ) et un snobisme grâce auquel il prétend s'intégrer dans l'élite parisienne. Cela le fait passer linguistiquement d'une manière permanente d'une langue régionale et provinciale à une langue parisienne soignée.

Au terme de cette réflexion, reste à parler du problème de la transposition que pose ce type d'œuvres, Illusions perdues en l'occurrence. Car l'œuvre de Balzac

\footnotetext{
4 Terme utilisé par Balzac In : Balzac, Honoré de. Illusions perdues. Paris : Ed. Gallimard, 2013.
} 
constitue un grand défi lors de la transposition en d'autres langues. II ne s'agit pas alors de restituer sémantiquement le discours des personnages, chose qui ne présente aucune difficulté, mais de restituer l'ensemble des connotations sociales, régionales, diachroniques..., qui sont attachées à la variation linguistique des personnages. La transposition doit alors être consciente de la variété des langages et des registres dans l'œuvre de Balzac, afin de restituer dans les langues d'arrivée un effet réel de vraisemblance équivalent à celui du texte d'origine.

Ainsi, il y a des termes et des tournures dont la transposition en d'autres langues présente une véritable difficulté. C'est ce qui se produit, par exemple, avec des termes et des expressions que nous entendons dans la bouche des personnages comme: septembriseur, lorette, liard, brûler son vin, longchamp, désangoulêmer, verse, lévite, poétriau...

\begin{tabular}{|c|c|c|}
\hline Le mot balzacien & La signification ${ }^{5}$ & La nature \\
\hline Septembriseur & $\begin{array}{l}\text { Un néologisme à marquage diachronique paru à cette époque } \\
\text { pour désigner ceux qui avaient participé au massacre des } \\
\text { royalistes détenus dans les prisons de Paris en septembre } 1792 \text {. }\end{array}$ & $\begin{array}{l}\text { Diachronisme } \\
\text { datant de } 1792\end{array}$ \\
\hline Lorette & $\begin{array}{l}\text { Un terme à marquage régional et diachronique à la fois, qui } \\
\text { désignait des demi-mondaines qui habitaient dans le quartier de } \\
\text { Notre-Dame de Lorette, peuplé à l'époque d'écrivains et d'artistes. } \\
\text { Le mot apparait en } 1830 \text {. }\end{array}$ & $\begin{array}{l}\text { Régionalisme e } \\
\text { diachronisme } \\
\text { de } 1830\end{array}$ \\
\hline Brûler son vin & $\begin{array}{l}\text { Une expression qui nous situe sur la région de Cognac. C'est « le } \\
\text { distiller pour le transformer en eau de vie ». }\end{array}$ & Régionalisme \\
\hline Désangoulêmer & $\begin{array}{l}\text { Se débarrasser des manières et des comportements d'Angoulême. } \\
\text { Désangoulêmé se dit d'un provincial qui s'est débarrassé de ses } \\
\text { manières provinciales et parait parisien. }\end{array}$ & Régionalisme \\
\hline Longchamp & $\begin{array}{l}\text { La promenade que les parisiens faisaient pendant la semaine } \\
\text { sainte aux Champs Elysées sur le terrain de l'ancienne abbaye de } \\
\text { Longchamp. }\end{array}$ & Régionalisme \\
\hline Lévite & Le nom méridional d'une redingote & Régionalisme \\
\hline Broum & $\begin{array}{l}\text { Onomatopée beaucoup utilisée par les enfants, en jouant, pour } \\
\text { imiter le bruit d'un moteur sur le pied d'accélérer. }\end{array}$ & $\begin{array}{l}\text { Onomatopée } \\
\text { française }\end{array}$ \\
\hline
\end{tabular}

${ }^{5}$ Toutes ces définitions sont prises dans l'édition annotée de Jacques Noiry de : Balzac, Honoré de, Illusions perdues, Ed. Gallimard, Paris, 2013; et vérifiées dans le dictionnaire Littré et le Trésor de langue française informatisé TLFi. Disponibles sur: http://atilf.atilf.fr/ et https://www.littre.org/ . Consultés le 11 décembre 2019. 


\begin{tabular}{|l|l|l|}
\hline Gondole & $\begin{array}{l}\text { Siège à la mode sous la Restauration, caractérisé par ses formes } \\
\text { recourbées et enveloppantes, mais ayant aujourd'hui un autre sens }\end{array}$ & Diachronisme \\
\hline Musico & $\begin{array}{l}\text { Lieu public dans les Pays-Bas où le peuple va boire, fumer, } \\
\text { entendre de la musique, etc. }\end{array}$ & Régionalisme \\
\hline Poétriau & $\begin{array}{l}\text { Terme familier et populaire créé sur « poétereau » qui signifie « un } \\
\text { mauvais et médiocre poète » }\end{array}$ & $\begin{array}{l}\text { Barbarisme } \\
\text { familier }\end{array}$ \\
\hline
\end{tabular}

La complexité sémantique et morphologique de ces termes, leurs connotations populaires et régionales ou diachronique, rendent très difficile une transposition adéquate en d'autres langues. En outre, il y a des termes qu'on ne peut transposer en un seul terme. Chose qui pousse les traducteurs à faire appel à des techniques de compensation comme les périphrases, les interventions métalinguistiques, les notes en bas de la page. Une autre difficulté réside, par exemple, dans la transposition du discours de Kolb :

-On m'ovrirait pien tes millions, queu cheu ne tirais bas une motte! Est-ce que che nei gonnais boind la gonzigne milidaire? $-U i$, fit l'Alsacien, chesbère edre assez riche ein chour pire lui domper sire le gazaquin, à ced ôme te chistice ! Ch'aime bas sa visache ! ${ }^{6}$ (BALZAC, 2013, p. 659).

Comme nous l'avons vu, le défi de la transposition, face au discours de Kolb, n'est pas tant d'en restituer le sens, mais de l'adapter phonétiquement, pour que le lecteur de l'œuvre transposée perçoive clairement cet accent alsacien de Kolb. La transposition doit restituer alors ce trait essentiel de l'idiolecte de ce personnage, sinon elle risquerait de lui ôter l'un de ses traits caractéristiques essentiels.

Pour conclure, la transposition de l'œuvre de Balzac en d'autres langues, présente une grande difficulté, vu sa nature polyphonique et hétéroglossique. Pour être fidèle au texte d'origine, le traducteur doit restituer cette spécificité, outil essentiel du réalisme balzacien et son but ultime, pour offrir au lecteur le reflet fidèle de cette nature hétéroglossique dans toutes ses formes régionale, populaire...

\section{Conclusion}

Pour que notre étude ne soit pas gratuite et fortuite, nous avons problématisé dans cette étude ce phénomène d'hétéroglossie littéraire pour rendre compte de l'incorporation

\footnotetext{
${ }^{6}$ L'italique par Balzac
} 
de la variation linguistique dans Illusions perdues. Textualisation que nous avons interprétée dans différentes perspectives. Ainsi, étant un des grands représentants du réalisme du XIXe siècle, Balzac, pour représenter sa société dans toutes ses dimensions, prête une grande importance aux langages de ses personnages. Pour ce faire, il sollicite tous les langages de son temps qu'il s'efforce de reproduire le plus fidèlement possible. II les met ainsi dans la bouche des personnages auxquels il délègue la parole. Ces derniers sont ainsi un facteur principal de stratification du langage du roman et la voie par laquelle l'hétéroglossie pénètre dans le roman. Ce réalisme linguistique, comme nous l'avons nommé, contribue au concept général de la mimésis aristotélicienne et accroît la vraisemblance de l'histoire. En effet, pour décrire sa société caractérisée par sa diversité linguistique, Balzac se voit contraint de se forger une langue hétérogène et hybride, capable de rendre compte de la mixité linguistique de son époque. Aussi la situation linguistique de l'époque de Balzac côtoyant plusieurs dialectes le pousse-t-elle à cette hétéroglossie littéraire. Par cette pratique, l'auteur inaugure une nouvelle esthétique d'écriture à un moment où les écrivains recouvrent encore la parole de leurs personnages d'une langue littéraire classique et soutenue. Esthétique qui demeure indissociable à l'art du roman et que certains voient comme la marque symptomatique de la modernité.

L'incorporation de la variation linguistique dans Illusions perdues n'est pas anodine et fortuite. Cette pratique, au contraire, remplit au sein de l'œuvre plusieurs fonctions. La première fonction qu'elle remplit est référentielle et réaliste, dans la mesure où la reproduction fidèle des parlers des personnages nous permet d'appréhender la situation linguistique de cette époque dans sa diversité réelle, et ancre l'histoire dans son contexte historique. Par cette pratique, Balzac donne un tour de vis supplémentaire au réalisme du XIXe siècle. Cette littérarisation de la variation au sein de l'œuvre joue un rôle sociolinguistique très important. Elle permet de renvoyer chaque locuteurpersonnage à son appartenance quelle qu'elle soit, à partir de sa manière de parler. Cette dernière s'offre au lecteur comme un marqueur sociolinguistique permettant de renseigner sur le personnage. Par-là, l'auteur manifeste aussi son attitude envers la politique linguistique de son époque. Son recours à la variation linguistique au sein de son roman est un moyen subtil pour dissimuler ses intentions derrière le langage de ses personnages et pour réhabiliter ainsi les dialectes de son temps, longtemps réprimés et abandonnés par l'Etat, en faisant surgir la voix des majorités silencieuses de son époque. Là où se manifeste la dimension idéologique de la langue balzacienne. 
Quoi qu'il en soit, la textualisation de la variation linguistique ne passe pas sans problèmes, et ce à bien des égards. D'une part, la littérarisation de la variation linguistique dans le roman diminue la littérarité de l'œuvre en raison de la discontinuité qu'elle produit dans la trame narrative. En effet, elle fait du roman une simple séquence dialogique en dialectes, privée de toute élaboration linguistique et de toute beauté de style ou du langage. D'autre part, la dimension diaphasique de la variation neutralise parfois la fonction sociolinguistique que l'hétéroglossie littéraire peut remplir. Car ces variétés linguistiques ne sont pas toujours un marqueur sociolinguistique pertinent. Un autre problème se pose lors de la transposition d'Illusions perdues en d'autres langues, vu sa nature plurilingue et hétéroglossique. En effet, devant cette spécificité, le traducteur n'est pas appelé seulement à restituer le sens de l'œuvre mais à restituer cette nature plurilingue dans toutes ses connotations sociolinguistiques, élément essentiel du réalisme balzacien. Tâche qui n'est pas aisé pour autant, voire impossible parfois.

Au terme de ce travail, nous pouvons dire que Balzac a réussi à inventer une nouvelle langue d'écriture qui vise à renouveler la langue littéraire classique. Une langue qui se donne au lecteur comme un amalgame de langages sans souci d'homogénéité ou de beauté, sur laquelle se fonde dans une grande partie le projet réaliste de l'auteur. Ce dernier tente de faire d'lllusions perdues une littérature nationale sans recourir nécessairement à la langue nationale. Son œuvre nous mène à repenser la relation entre la littérature, la langue et la société. Une piste encore fertile qui doit être explorée par des études à venir à travers des perspectives très diverses.

\section{Bibliographie}

AUERBACH, E. Mimésis : la représentation de la réalité dans la littérature occidentale. Traduit de l'allemand par Cornélius Heim. Paris: Gallimard, 1968.

BAKHTINE, M. Esthétique et théorie du roman. Traduit du russe par Daria Olivier. Paris: Gallimard, 1978.

BALZAC, H. Illusions perdues. Paris: Ed. Gallimard, 2013.

BALZAC, H. Madame Firmiani. Paris: Tome II, 1832.

BARTHES, R. Roland Barthes par Roland Barthes. Paris: Éd. Seuil, 1975.

BARTHES, R ; BERSANI, L ; HAMON, P ; RIFFATERRE, M; WATT, I. Littérature et réalité. Paris: Ed. Seuil, 1982. 
DUFOUR, P. Illusions perdues : une histoire des mœurs langagières. L'information littéraire 1/2004 (Vol. 56), p. 14-23.

HAMON, P. Voir la ville, Romantisme N²83, 1994.

HAMON, P. Un discours contraint. Poétique N¹6 : 411-445, 1973.

GADET, F. La variation sociale en français. Paris: Ophrys, 2007.

GAUTIER, T. Honoré de Balzac (1859). Repris partiellement en préface à La Comédie humaine (édition de 1868), in : Balzac (Stéphane Vachon éditeur), Presses de l'université de Paris-Sorbonne, « Mémoire de la critique », 1999.

GAUVIN, L. Les langues du roman. Du plurilinguisme comme stratégie textuelle. Montréal: PU de Montréal, 1999.

GAUVIN, L. In: Introduction à une Poétique du Divers. Montréal, Presses de I'Université de Montréal, « Prix de la revue Études françaises », 1995. Paris: Gallimard, 1996.

GRUTMAN, R. Des langues qui résonnent. L'hétérolinguisme au XIXe siècle québécois. Montréal: Fides/Cétuq, 1997.

JAKOBSON, R. Questions de poétique. Paris: Ed. Seuil, 1973.

LABOURET, D; MEUNIER, A. Méthodes $d u$ français. Paris: Bordas, 2004. 